\title{
The puzzling Maia candidate star $\alpha$ Draconis
}

\author{
Th. Kallinger ${ }^{1}$, I. Iliev ${ }^{2}$, H. Lehmann ${ }^{3}$ and W.W. Weiss ${ }^{1}$ \\ ${ }^{1}$ Department of Astronomy, University of Vienna, Türkenschanzstrasse 17, A-1180, Vienna, \\ Austria, \\ email: lastname@astro.univie.ac.at \\ ${ }^{2}$ National Astronomical Observatory Rozhen, Bulgaria, \\ email: iliani@libra.astro.bas.bg \\ ${ }^{3}$ Thüringer Landessternwarte Tautenburg, Tautenburg, Germany, \\ email:lehm@tls-tautenburg.de
}

\begin{abstract}
The existence of Maia variables has been in dispute since 1955. They are supposed to be located between the blue edge of the classical instability strip and the red border of the slowly pulsating $B$ stars, hence in a domain of the HRD where no excitation mechanism for pulsation is yet known. But luminosity variations were discovered in time series of $\alpha$ Draconis, an A0II Maia candidate star, with a period of about 53 minutes and an amplitude of less than $0.002 \mathrm{mag}$. Spectroscopic time series indicate radial velocity variations with the same period and an amplitude of about $40 \mathrm{~m} \mathrm{~s}^{-1}$. Alpha Dra is a single-lined spectroscopic binary with an orbital period of 51.4 days and a distance between the components of about $0.46 \mathrm{AU}$. Tidal interaction may therefore be responsible for pulsation. If true, the pulsation amplitude should be modulated with the orbit as is indeed indicated by recent observations.
\end{abstract}

Keywords. Stars: oscillations (including pulsations), stars: individual: ( $\alpha$ Dra)

\section{Introduction}

Current theory does not predict pulsating stars between the blue edge of the $\delta$ Scuti instability strip and the red edge of the slowly pulsating B stars (SPB). No opacity anomaly is known like the metal opacity bump for the SPB stars or the He II bump for the $\delta$ Scuti stars which could drive pulsation. However, Struve (1955) introduced a group of variable stars with spectral types between B7V-III and A2V-II and pulsation periods between 2 and 8 hours. He called them Maia stars, but Struve himself later could not confirm pulsation in Maia. Their existence still is debated (Percy \& Wilson 2000 and references therein). Pulsation of a Maia type star proven beyond doubt would pose a serious problem for theoretical astrophysics. Either a new opacity source has to be identified or a yet unknown pulsation mechanism.

\section{Observations}

Indications for variablility in the Maia candidate star $\alpha$ Dra observed during a photometric monitoring campaign with a twin APT $\dagger$ in the winter of 2001 motivated us to investigate this $3.6 \mathrm{~V}$ A0III star. In June, 2002, 165 sets of photometric data were obtained with the APT during 17 nights. The Fourier spectrum (Fig. 1) shows a peak at about $27 \mathrm{~d}^{-1}$ (a period of about $53 \mathrm{~min}$ ) with an amplitude of about $1.4 \mathrm{mmag}$, but with poor $S / N$. This frequency was confirmed by Hipparcos photometry with an even better $S / N$. As is discussed by Kallinger \& Weiss (2002), Hipparcos has a good detection

$\dagger$ Wolfgang-Amadeus: the University of Vienna twin automatic photoelectric telescope in Washington Camp, Arizona (Strassmeier et al. 1997) 


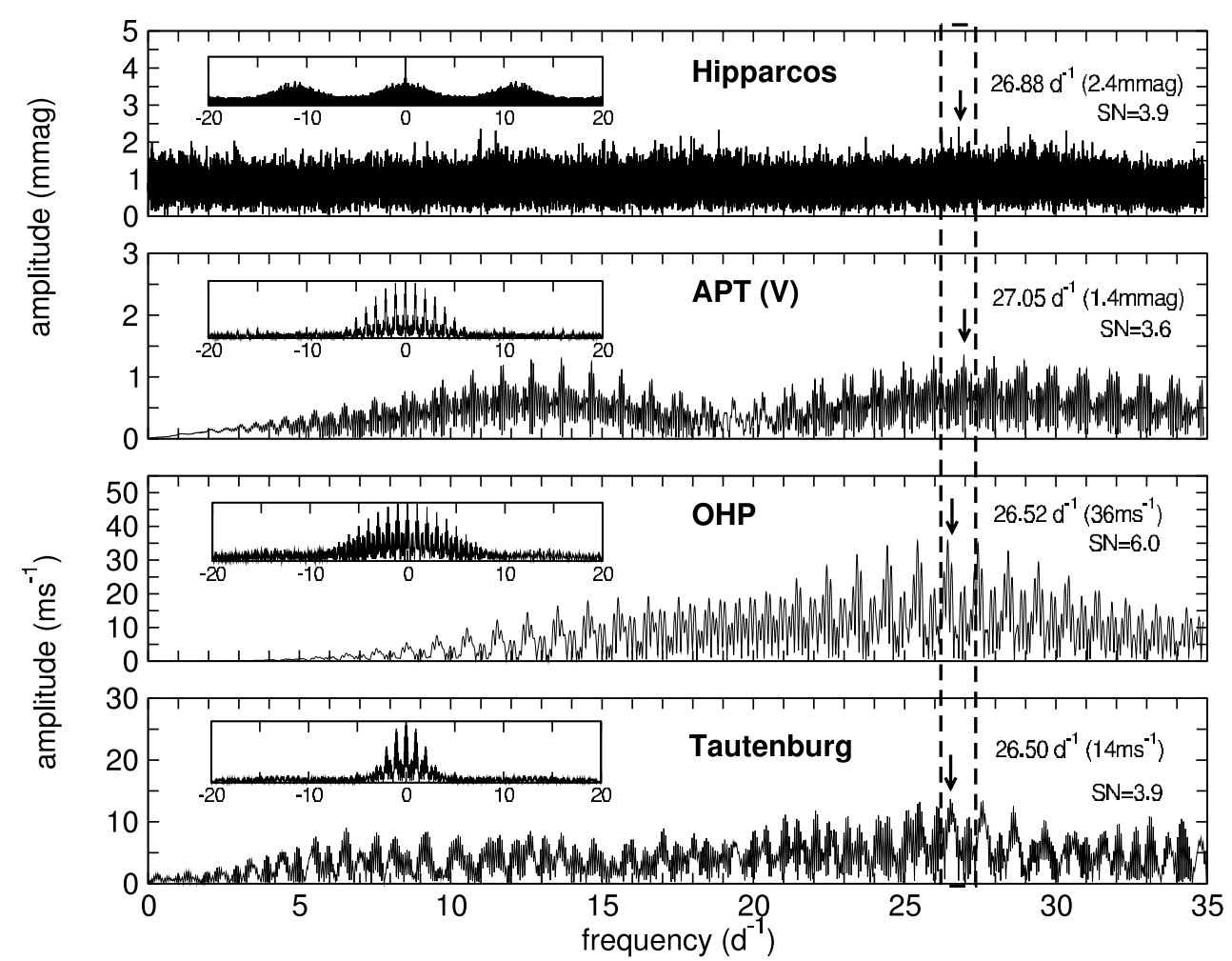

Figure 1. Fourier analysis of different photometric and spectroscopic time series of $\alpha$ Dra. The Fourier spectra were derived from following data. From top to bottom: Hipparcos epoch photometry, APT in Johnson $V$, spectral time series obtained at OHP and at the Thüringer Landessternwarte Tautenburg. The inserted panels show the corresponding spectral windows.

capability in the 15 to $35 \mathrm{~d}^{-1}$ frequency range, whereas frequencies around $11 \mathrm{~d}^{-1}$ are likely of instrumental origin.

In January, 2003, 140 echelle spectra were obtained during 10 nights at the Observatiore de Haute-Provence $(\mathrm{OHP})$ from $\lambda 3900$ to $\lambda 6800$ with $\mathrm{R} \approx 35000$. Periodic $\mathrm{RV}$ variations with $26.5 \mathrm{~d}^{-1}$ and an amplitude of about $36 \mathrm{~m} \mathrm{~s}^{-1}$ were detected in the residuals to the orbit using a technique described by Bouchy et al. (2001). In February, 2004, 17 spectra (centered on CaI $\lambda 6456.2$ and Fe II $\lambda 5362.7$ ) were obtained during one night with the Bulgarian 2.0-m RCC Telescope at Rozhen. Periodic RV variations determined from the line center (Fig .2) confirm the photometric frequency, but with a 3 times higher amplitude.

Tidal interaction in the close binary system $\alpha$ Dra might excite pulsation with an amplitude depending on the orbital phase. The first evidence encouraged us to organise a spectroscopic campaign with two main goals: the redefinition of the orbital parameters (Lehman \& Scholz 1993) and time resolved spectroscopy at different orbit phases. The campaign at the Thüringer Landessternwarte Tautenburg started last summer and is still ongoing. Now the entire orbit is covered with spectra ranging from $\lambda 4750$ to $\lambda 7070$ and with $\mathrm{R} \approx 67000$. Further 1150 time resolved spectra were acquired during 45 nights around apastron. The Fourier spectrum of the RV time series (Fig. 1) shows a peak at about $26.5 \mathrm{~d}^{-1}$ with an amplitude of about $14 \mathrm{~m} \mathrm{~s}^{-1}$ which is significantly lower than that determined from the OHP spectra. 


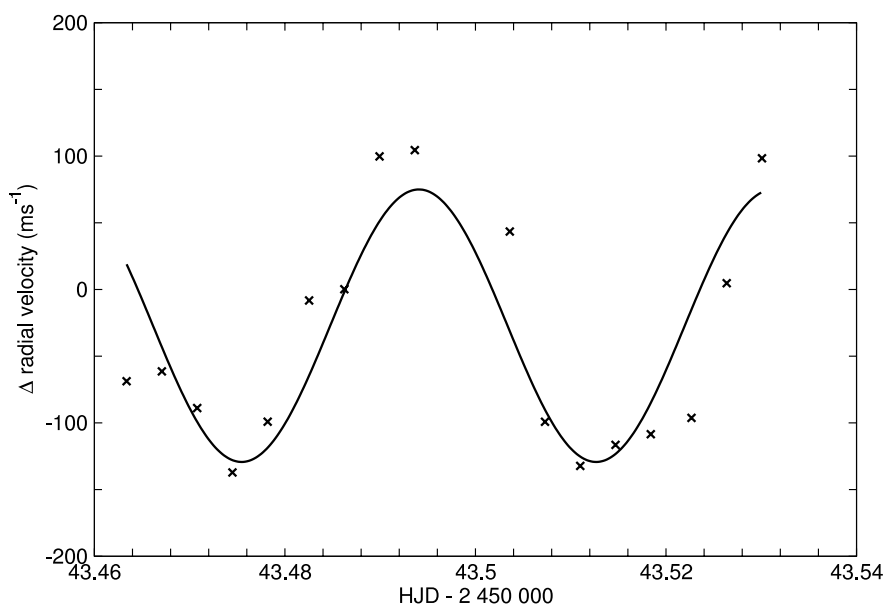

Figure 2. Line center variations (in $\mathrm{m} \mathrm{s}^{-1}$ ) of two spectral lines (Ca I $\lambda 6456.2$ and Fe II $\lambda 5362.7$ ) versus time for a set of 17 spectra obtained during one night at Rozhen. The line represents the best sinusoidal fit with a frequency of $26.88 \mathrm{~d}^{-1}$ resulting in a three times higher amplitude $\left(105 \mathrm{~m} \mathrm{~s}^{-1}\right)$ than determined from OHP spectra.

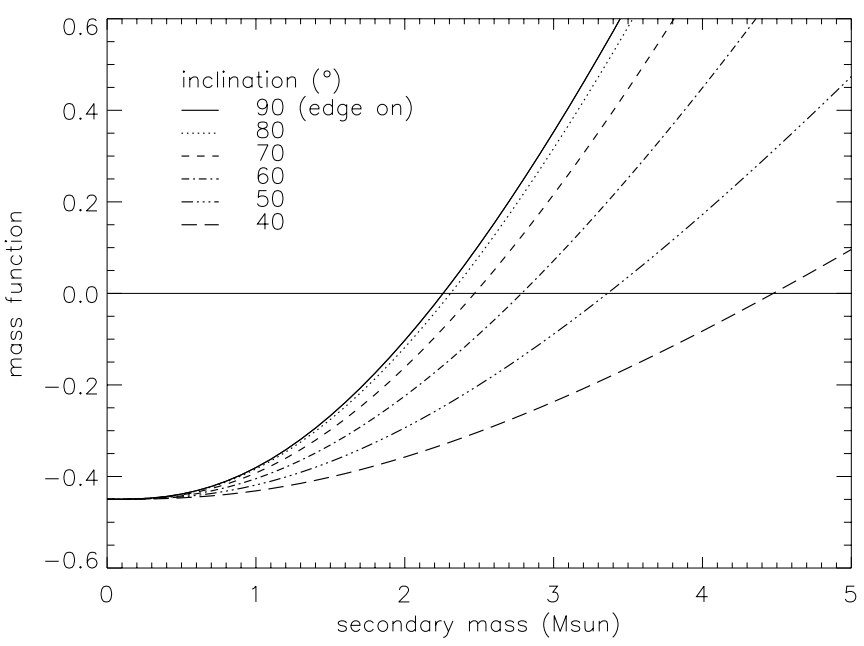

Figure 3. Gradient of the mass function for the $\alpha$ Dra binary system for different inclinations with a primary mass of $2.8 M_{\odot}$. The secondary mass follows from the zero point of a curve given by the inclination. Assuming a nearly edge on system the lowest possible secondary mass is about $2.2 M_{\odot}$.

\section{Binary system}

$\alpha$ Dra is a single-lined spectroscopic binary system with the orbital parameters listed in Table 1. Fundamental parameters (Adelman et al. 2001) and evolutionary tracks indicate a primary mass of $2.8 \pm 0.2 M_{\odot}$ (see Fig. 5 ). The secondary mass has to be sufficiently smaller, otherwise we would detect a secondary spectrum. The lower mass limit is given by the zero point of the mass function (see Fig. 3) which indicates $2.2 M_{\odot}$ for an inclination of about $90^{\circ}$. Hence both stars lie outside any known instability region. Assuming such a mass for the secondary the semi-major axis of the binary system is about $0.46 \mathrm{AU}$. The secondary RV curve is given in (Fig. 4). 


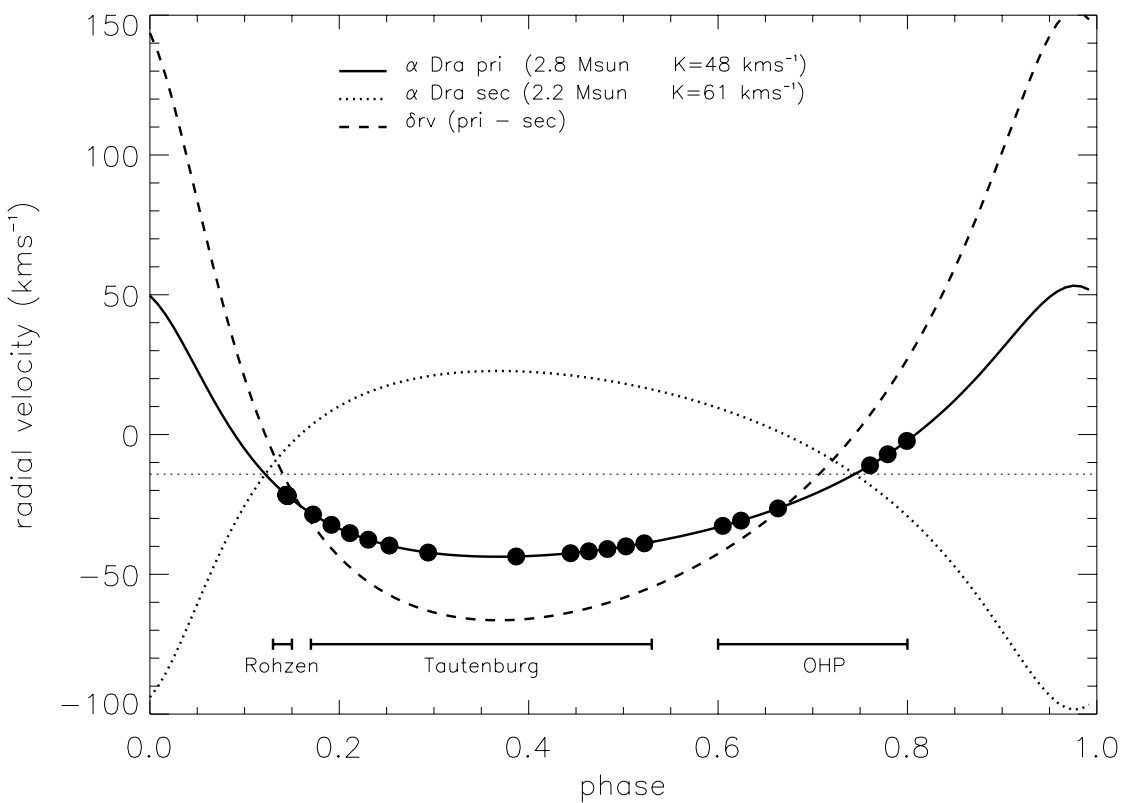

Figure 4. RV curve of both components of the $\alpha$ Dra binary system. The dots indicate orbit phases when the spectral time series were obtained.

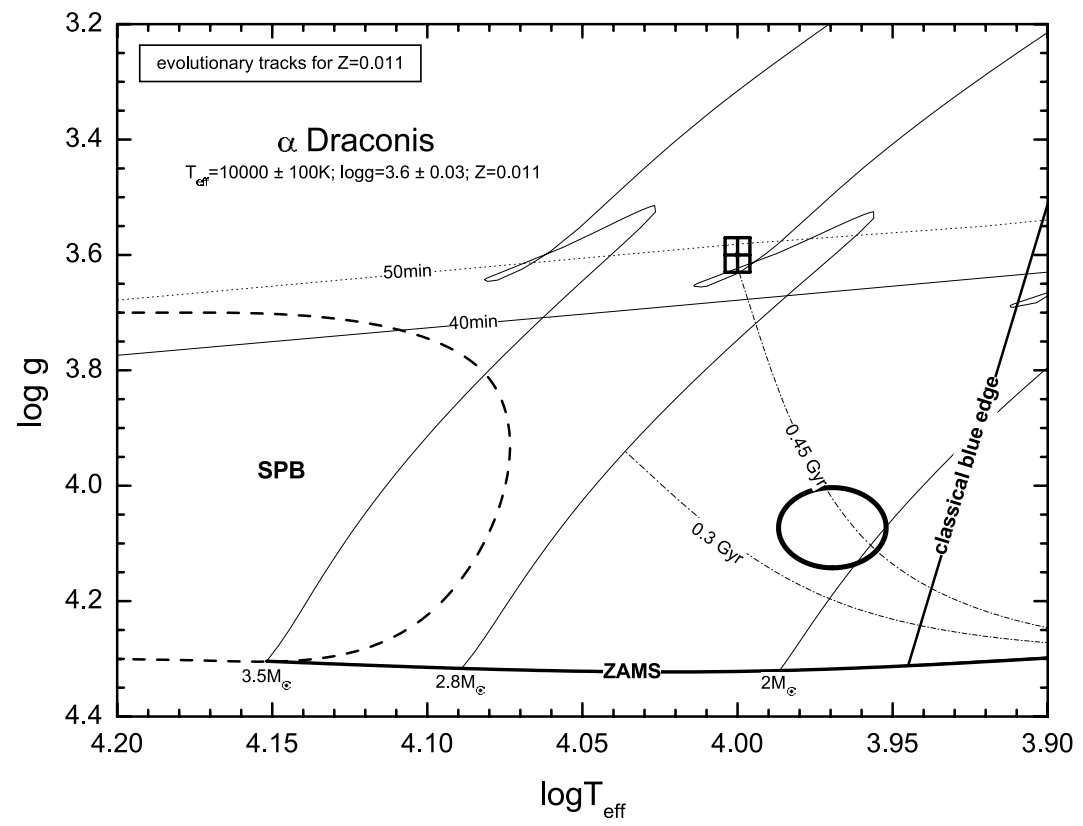

Figure 5. HR-diagram showing the error box of $\alpha$ Dra. The evolutionary tracks correspond to 2, 2.8 and $3.5 M_{\odot}$. The ellipse indicates the region where we expect the secondary (based on equal age and our mass estimate). The two lines marked with $40 \mathrm{~min}$ and $50 \mathrm{~min}$ are lines of equal frequency of maximum power, extrapolated from the solar 5 min oscillation (Kjeldsen \& Bedding 1995). 
Table 1. Orbital parameters of the binary system $\alpha$ Dra (errors of the last two digits in parenthesis).

\begin{tabular}{lrl}
\hline$P$ & $51.439(14)$ & $(\mathrm{d})$ \\
$K$ & $48.488(80)$ & $\left(\mathrm{km} \mathrm{s}^{-1}\right)$ \\
$R V_{0}$ & $-14.17(22)$ & $\left(\mathrm{km} \mathrm{s}^{-1}\right)$ \\
$e$ & $0.4219(16)$ & \\
$T$ & $2452881.736(51)$ & \\
$\omega$ & $22.11(29)$ & $\left(^{\circ}\right)$ \\
\hline
\end{tabular}

\section{Conclusions}

We have fascinating photometric AND spectroscopic evidence for variability in the Maia candidate star $\alpha$ Dra. The observations show different amplitudes at different orbital phases. This suggests tidal interaction as the pulsation driving mechanism. Further observations near periastron are required to check the trend of pulsation amplitude with orbit phase. The secondary's signature is marginally visible in our spectroscopic data what is compatible with simulations based on a high $v \sin i$ of about $100 \mathrm{~km} \mathrm{~s}^{-1}$ for the companion.

\section{Acknowledgements}

Financial support was received from the Bundesministerium für Bildung, Wissenschaft und Kultur (project EXTRACTOR) and the Austrian Fonds zur Förderung der wissenschaftlichen Forschung (project P14984).

\section{References}

Adelman, S. J., Caliskan, H., et al. 2001, A\&\& 371, 1078

Bouchy, F., Pepe F., Queloz, D. 2001, A\& $A$ A 374, 733

Elst, E. W., Nelles, B. 1983, A\&AS 53, 215

Kallinger, Th., Weiss, W. W. 2002, A\&A 385, 533

Kjeldsen, H., Bedding, T. R. 1995, A\&SA 293, 87

Lehmann, H., Scholz, G. 1993, ASP Conf. Series V.44

Percy, J. R., Wilson, J. B. 2000, PASP 112, 84

Strassmeier, K. G., Boyd, L. J. et al. 1997, PASP 109, 697

Struve, O. 1955, Sky and Telescope 14, 461 